\title{
Qubit authentication
}

\author{
Marcos Curty, f David J. Santos, 间 and Esther Pére用 \\ ETSIT, Universidad de Vigo, Campus Universitario s/n, E-36200 Vigo (Spain) \\ Priscila García-Fernández \\ Instituto de Óptica, CSIC, Serrano, 123, E-28006 Madrid (Spain)
}

(Dated: October 25, 2018)

\begin{abstract}
Secure communication requires message authentication. In this paper we address the problem of how to authenticate quantum information sent through a quantum channel between two communicating parties with the minimum amount of resources. Specifically, our objective is to determine whether one elementary quantum message (a qubit) can be authenticated with a key of minimum length. We show that, unlike the case of classical-message quantum authentication, this is not possible.

PACS numbers: 3.67.Dd, 03.67.Hk, 03.67.Lx.
\end{abstract}

\section{INTRODUCTION}

Cryptography deals about communication in the presence of adversaries [1], and one of its fundamental goals is to provide message authentication (also called dataorigin authentication) [2]. This is a process whereby a party is corroborated as the original source of some specified data created, transmitted, or stored at some time in the past. To assure message authentication, one then must have the ability to detect any data manipulation by unauthorized parties. This is particularly important in public-key cryptography, in which users must be confident about the authenticity of the public-keys of the partners involved in the communication.

Classical cryptography provides data-origin authentication by means of two general techniques: message authentication codes (MACs) [3], and digital-signature schemes [П]. In both cases, the authentication process is specified by two algorithms: an encoding, or tagging algorithm (possibly stochastic), and a decoding or verification algorithm. When the sender (Alice) wishes to send a certified message to a recipient (Bob), she generates, employing the encoding algorithm, an authentication tag or a signature (in both cases a function of the message and a secret encoding-key) and appends it to the message before actually sending it. Notice that this tagged-message may be sent in the clear; the authentication problem is, therefore, very different to the one associated to encryption since no secrecy is necessary for secure message authentication. On the reception side, Bob verifies the authenticity of the message by means of the specified decoding procedure, which depends on the message, the tag, and a decoding-key. This algorithm returns a bit indicating when Bob must regard the mes-

\footnotetext{
*mcurty@com.uvigo.es

+ dsantos@com.uvigo.es

estherperez@edu.xunta.es

$\S$ pgarcia@foton0.iem.csic.es
}

sage as authentic, and accept it as coming from Alice, and when he must discard it. The basic requirement is that the tags, which are produced by the encoding algorithm, be accepted as valid by the verification algorithm when a decoding-key corresponding to the encoding-key is used on the tagging procedure. When a message authentication scheme fulfills this requisite it is said that it provides perfect deterministic decoding.

Choosing between MACs and digital-signature schemes depends on the communication context. Typically, MACs are employed when the receiver is predetermined at the time of message transmission, and the decoding-key he owns, which can be equal to the encoding-key, is secret. In signature schemes, on the contrary, the decoding-key is public, and therefore known also to the adversary (Eve); this fact guarantees that anybody can verify the authenticity of data (universal verification), and that Alice cannot later repudiate having signed the message, since no one but she owns the encoding-key.

Although several information-theoretic secure MACs have been proposed (see, e.g., 30), the security provided by signature schemes depends on unproven assumptions related to the intractability of certain difficult mathematical problems, such as the prime-factorization of large numbers $[5]$ or the discrete-logarithm computation [6]. Unfortunately, if a quantum computer is ever built, Shor's quantum algorithms [7] could break classical signature schemes easily, i.e., in polynomial time.

The use of quantum resources in user authentication has been proposed before in QKD scenarios [8, 9]. More recently, several proposals of general message authentication using quantum resources have been made. Gottesman and Chuang [10] have proposed a quantum version of classical digital-signature schemes. Their technique requires limited circulation of the public key, but it allows unconditionally secure authentication of classical messages. A disadvantage of this protocol, as stated by the authors, is that it requires several non-reusable key-bits for each message-bit signed. Leung [11], in another recent paper, also studies quantum message authentication. 
Her protocol requires a two-way classical channel between partners, thus making the overall security dependent on the security of that channel. In a previous article 12] some of us proposed a class of quantum authentication protocols that allow secure authentication of classical binary messages with one bit as the authentication key. This improves the efficiency of classical MACs, that require, for the authentication of binary messages, at least two-bit keys. In this paper we adapt this class of protocols to the authentication of quantum messages (qubits), and study its security. We show that, using the amount of quantum resources needed to authenticate one-bit classical messages, the intrinsic nature of quantum information makes it impossible to detect every unauthorized quantum data manipulation by a potential adversary, thus making qubit authentication not possible.

The paper is organized as follows. In Section II we describe a general class of one-qubit message authentication protocols. This class can be seen as a generalization of the one presented in 12 to authenticate classical binary messages. In Section III we analyze the security of these protocols against several attacks. First, we analyze the no-message attack, in which the sender has not initiated the transmission (there is no message in the channel), and Eve attempts to prepare a fake quantum message with the intention of passing Bob's verification test. Then, we study more subtle attacks, those in which Eve has access to what is transmitted. After studying all these attacks we show that it is not possible to keep the failure probability of the authentication protocol below one.

\section{QUANTUM MESSAGE AUTHENTICATION}

Suppose Alice needs to send a certified quantum message to Bob. The goal is to make Bob confident about the authenticity of the message and sender. For the sake of simplicity, let us assume that the state to send certified is an arbitrary qubit described by the density operator $\rho_{\mathcal{M}}$ operating on some two-dimensional message space $\mathcal{M}$. This qubit may be locally generated by her, or she may be acting as a relay station between two other parties. In order to certify this message, Alice follows the standard procedure in classical authentication: She appends a tag to the message in such a way that the recipient, Bob, may verify the tag and so convince himself about the identity of the message originator. The difference with respect to the classical case is that now the tag is also a quantum state, given by the density operator $\rho_{\mathcal{T}}$, of some tag space $\mathcal{T}$. Therefore, the quantum taggedmessage is described by the operator $\rho_{\mathcal{E}}=\rho_{\mathcal{M}} \otimes \rho_{\mathcal{T}}$ that acts on the state space $\mathcal{E}=\mathcal{M} \otimes \mathcal{T}$. Since our objective is to determine whether authentication is possible with the minimum amount of resources, we shall regard $\mathcal{T}$ as a two-dimensional space. Although this might seem a strong restriction, it can be shown that having a bigger tag does not improve the security of the protocol, at least when dealing with an ideal, error-free quantum channel, as is the case considered here. In a noisy channel, a bigger tag space would certainly be useful in the detection of errors in the channel that might alter the message, in the way Quantum Error Correcting Codes (QECC) usually work. We shall return to this point with more detail later, at the end of Section III. Finally, since we are interested in perfect deterministic decoding, valid and invalid tags must belong, respectively, to orthogonal subspaces in $\mathcal{T}$, but, since one cannot find orthogonal mixed states in a two-dimensional space $(\mathcal{T}), \rho_{\mathcal{T}}$ must be a pure state, namely $\rho_{\mathcal{T}}=|0\rangle\left\langle\left. 0\right|_{\mathcal{T}}\right.$.

No authentication is possible without a previously shared secret between the two communicating parties. This key, which may have been exchanged directly or by means of a trusted third party (a certification authority), can be a classical or a quantum one. In our proposal we shall assume that Alice and Bob share a maximally entangled quantum state as their secret authentication key. For instance, each of the parties could own one qubit of a publicly-known singlet state $|\psi\rangle_{A B}=$ $\frac{1}{\sqrt{2}}\left(|01\rangle_{A B}-|10\rangle_{A B}\right)$. It can be argued that, in a realistic scenario, dealing with classical keys would be more advantageous. In fact, our protocol can equally operate with one-bit classical keys. However, we prefer the use of quantum keys for their better key-management properties - no copying of the key remains undetected if extra quantum key is included for this check.

The main difference between authenticating a classical message and a quantum one resides in the nature of $\rho_{\mathcal{M}}$. If $\rho_{\mathcal{M}}$ is a quantum message, it belongs to the continuous space of density operators acting on $\mathcal{M}$. Since Alice may act just as a relay station, we shall assume that $\rho_{\mathcal{M}}$ is unknown to her. In the classical case, there are only two classical messages to send, ' 0 ' or ' 1 '. To encode them unambiguously Alice and Bob need to agree on a particular orthonormal basis of $\mathcal{M},\left\{|0\rangle_{\mathcal{M}},|1\rangle_{\mathcal{M}}\right\}$, so $\rho_{\mathcal{M}}$ would be restricted to be either $|0\rangle\left\langle\left. 0\right|_{\mathcal{M}}\right.$ or $\left.\mid 1\right\rangle\left\langle\left. 1\right|_{\mathcal{M}}\right.$. This decision can be made openly, adding no more secrecy between the parties.

According to the notation introduced above, the state of the global system (key+tagged-message) is given by

$$
\rho_{A B \mathcal{E}}=|\psi\rangle\left\langle\left.\psi\right|_{A B} \otimes \rho_{\mathcal{E}}=\mid \psi\right\rangle\left\langle\left.\psi\right|_{A B} \otimes \rho_{\mathcal{M}} \otimes \mid 0\right\rangle\left\langle\left. 0\right|_{\mathcal{T}} .\right.
$$

Next Alice performs, on her part of $|\psi\rangle_{A B}$ and on the tagged-message, an encoding operation $E_{A \mathcal{E}}$. We may write this unitary operation in the general form:

$$
E_{A \mathcal{E}}=|0\rangle\left\langle\left. 0\right|_{A} \otimes 1_{\mathcal{E}}+\mid 1\right\rangle\left\langle\left. 1\right|_{A} \otimes U_{\mathcal{E}},\right.
$$

where $U_{\mathcal{E}}$ is some arbitrary unitary quantum operation on $\mathcal{E}$. Basically, the action of $E_{A \mathcal{E}}$ is equivalent to the selection, triggered by the state of a one-bit key, of one operator from an arbitrary pair of publicly-known unitary ones (in our case, $1_{\mathcal{E}}$ and $U_{\mathcal{E}}$ ). Once this operator is selected, it is applied to $\rho_{\mathcal{E}}$ before sending it through the quantum channel. The reason to enforce the unitarity of 
these two operators is that it allows Bob to easily undo their action. But this is not the only way in which perfect deterministic decoding can be achieved. As shown in 13, 14, 15, more general quantum operations, such as trace-preserving completely-positive (TPCP) maps, can, under certain circumstances, be reversed, and, therefore, used in $E_{A \mathcal{E}}$ instead of $1_{\mathcal{E}}$ and $U_{\mathcal{E}}$. However, for simplicity in the formalism we limit ourselves to the case of unitary operators.

If we denote by $\rho_{A B \mathcal{E}}^{e}$ the density operator describing the state of the global system after the encoding operation, i.e., $\rho_{A B \mathcal{E}}^{e}=E_{A \mathcal{E}} \rho_{A B \mathcal{E}} E_{A \mathcal{E}}^{\dagger}$, then the state of the tagged-message that Alice sends to Bob through the quantum channel between them is given by $\rho_{\mathcal{E}}^{e}=$ $\operatorname{tr}_{A B}\left(\rho_{A B \mathcal{E}}^{e}\right)$, where $\rho_{A B \mathcal{E}}^{e}$, making use of (2), can be written as

$$
\begin{aligned}
\rho_{A B \mathcal{E}}^{e} & =\frac{1}{2}\left(| 0 1 \rangle \langle 0 1 | _ { A B } \otimes \rho _ { \mathcal { E } } + | 1 0 \rangle \left\langle\left.10\right|_{A B} \otimes U_{\mathcal{E}} \rho_{\mathcal{E}} U_{\mathcal{E}}^{\dagger}\right.\right. \\
& -|01\rangle\left\langle\left. 10\right|_{A B} \otimes \rho_{\mathcal{E}} U_{\mathcal{E}}^{\dagger}-\mid 10\right\rangle\left\langle\left. 01\right|_{A B} \otimes U_{\mathcal{E}} \rho_{\mathcal{E}}\right) .(3)
\end{aligned}
$$

From this expression, it is easy to obtain the taggedmessage in the channel:

$$
\rho_{\mathcal{E}}^{e}=\frac{1}{2}\left(\rho_{\mathcal{E}}+U_{\mathcal{E}} \rho_{\mathcal{E}} U_{\mathcal{E}}^{\dagger}\right) .
$$

On the reception side, Bob decodes the information sent by Alice performing the unitary decoding operation

$$
D_{B \mathcal{E}}=|0\rangle\left\langle\left. 0\right|_{B} U_{\mathcal{E}}^{\dagger}+\mid 1\right\rangle\left\langle\left. 1\right|_{B} 1_{\mathcal{E}}\right.
$$

on his qubit of the singlet and the tagged-message received: $\rho_{A B \mathcal{E}}^{d}=D_{B \mathcal{E}} \rho_{A B \mathcal{E}}^{e} D_{B \mathcal{E}}^{\dagger}$. Using (3) and (5) in this equation one can easily calculate the decoded taggedmessage as $\rho_{\mathcal{E}}^{d}=\operatorname{tr}_{A B}\left(\rho_{A B \mathcal{E}}^{d}\right)=\rho_{\mathcal{E}}$. Finally, Bob verifies the state of the tag: He performs the orthogonal measurement $\left\{|0\rangle\left\langle\left. 0\right|_{\mathcal{T}}, \mid 1\right\rangle\left\langle\left. 1\right|_{\mathcal{T}}\right\}\right.$ over the tag-portion of $\rho_{\mathcal{E}}^{d}$, where $|1\rangle_{\mathcal{T}}$ is the state in $\mathcal{T}$ orthogonal to the tag $|0\rangle_{\mathcal{T}}$. If the result of such a measurement is $|0\rangle_{\mathcal{T}}$, Bob should assume that no tampering has taken place, and therefore extract the quantum message sent to him; otherwise, he rejects the message received.

\section{SECURITY ANALYSIS}

In the previous section we have claimed that the proposed tag-based quantum authentication protocol provides perfect deterministic decoding. This means that the protocol would fail only if Bob accepted a message as an authenticated one when that is not the case (due to the unnoticed action of Eve). When dealing with forgery strategies we must consider two main types of attacks: The no-message attack, and the message attack [2]. The first one is the simpler: Before any message is sent by
Alice to Bob, Eve attempts to prepare a quantum state that passes the decoding algorithm. The message attack is more subtle and severe: Eve could access the authentic messages transmitted, and try to produce a forged message based on the information gained. In the following discussion we shall show how, unlike the case of authentication of classical messages [12], when dealing with a one-qubit message it is impossible to select a unitary operation $U_{\mathcal{E}}$ that makes all Eve's possible attacks unsuccessful. We find necessary conditions for the probability of successful forgery by a no-message attack (in section IIIA), and by a measurement attack (in section IIIB-1), to be less than one. Then in section IIIB-2 we show that under these conditions, there exists another attack, consisting of a unitary operator applied to the message and tag system, that succeeds with probability one.

\section{A. No-message attack}

Suppose that Eve prepares some quantum state $\rho_{\mathcal{E}}^{E} \in \mathcal{E}$ and sends it to Bob trying to impersonate Alice. When Bob receives this quantum message he cannot know that it comes from a forger, so he follows the procedure explained in the previous section: He performs the decoding operation $D_{B \mathcal{E}}$ and then an orthogonal measurement $\left\{|0\rangle\left\langle\left. 0\right|_{\mathcal{T}}, \mid 1\right\rangle\left\langle\left. 1\right|_{\mathcal{T}}\right\}\right.$ over the tag space. Before all this takes place, Bob 'sees', as the encoded global state, the density operator $\rho_{A B \mathcal{E}}^{e}=|\psi\rangle\left\langle\left.\psi\right|_{A B} \otimes \rho_{\mathcal{E}}^{E}\right.$. After Bob's decoding, the tagged-message is given by

$$
\rho_{\mathcal{E}}^{d}=\operatorname{tr}_{A B}\left(\rho_{A B \mathcal{E}}^{d}\right)=\frac{1}{2}\left(\rho_{\mathcal{E}}^{E}+U_{\mathcal{E}}^{\dagger} \rho_{\mathcal{E}}^{E} U_{\mathcal{E}}\right)
$$

where $\rho_{A B \mathcal{E}}^{d}=D_{B \mathcal{E}} \rho_{A B \mathcal{E}}^{e} D_{B \mathcal{E}}^{\dagger}$. As we have seen, Bob rejects the message if the result of his orthogonal measurement on $\mathcal{T}$ is $|1\rangle_{\mathcal{T}}$; therefore, the probability $P_{f}$ that Eve deceives Bob is:

$$
P_{f}=\left\langle 0\left|\operatorname{tr}_{\mathcal{M}}\left[\frac{1}{2}\left(\rho_{\mathcal{E}}^{E}+U_{\mathcal{E}}^{\dagger} \rho_{\mathcal{E}}^{E} U_{\mathcal{E}}\right)\right]\right| 0\right\rangle_{\mathcal{T}} .
$$

This quantity depends both on Eve's strategy - her selection of $\rho_{\mathcal{E}}^{E}$ - and on the quantum operation $U_{\mathcal{E}}$. She will succeed with probability one if she chooses a $\rho_{\mathcal{E}}^{E}$ that satisfies

$$
\operatorname{tr}_{\mathcal{M}}\left(\rho_{\mathcal{E}}^{E}\right)=|0\rangle\left\langle\left. 0\right|_{\mathcal{T}}\right.
$$

and

$$
\operatorname{tr}_{\mathcal{M}}\left(U_{\mathcal{E}}^{\dagger} \rho_{\mathcal{E}}^{E} U_{\mathcal{E}}\right)=|0\rangle\left\langle\left. 0\right|_{\mathcal{T}}\right.
$$

In order to check whether Eve succeeds, let us first consider the case in which she prepares a pure state 
$\rho_{\mathcal{E}}^{E}=|\phi\rangle\left\langle\left.\phi\right|_{\mathcal{E}}\right.$. The conditions (8) and (9) above can then be rewritten as

$$
\operatorname{tr}_{\mathcal{M}}\left(|\phi\rangle\left\langle\left.\phi\right|_{\mathcal{E}}\right)=|0\rangle\left\langle\left. 0\right|_{\mathcal{T}}\right.\right.
$$

and

$$
\operatorname{tr}_{\mathcal{M}}\left(U_{\mathcal{E}}^{\dagger}|\phi\rangle\left\langle\left.\phi\right|_{\mathcal{E}} U_{\mathcal{E}}\right)=|0\rangle\left\langle\left. 0\right|_{\mathcal{T}},\right.\right.
$$

respectively. From equation (10) we conclude that Eve should choose $|\phi\rangle_{\mathcal{E}}=|\psi\rangle_{\mathcal{M}} \otimes|0\rangle_{\mathcal{T}}$, with $|\psi\rangle_{\mathcal{M}}$ any pure state in $\mathcal{M}$. When this result is used in (11), then

$$
U_{\mathcal{E}}^{\dagger}\left(|\psi\rangle_{\mathcal{M}} \otimes|0\rangle_{\mathcal{T}}\right)=|\omega\rangle_{\mathcal{M}} \otimes|0\rangle_{\mathcal{T}}
$$

should also be satisfied, where $|\omega\rangle_{\mathcal{M}}$ is some pure state in $\mathcal{M}$.

Without loss of generality, let us write $U_{\mathcal{E}}$ in the form

$$
U_{\mathcal{E}}=\sum_{i, j=0}^{1} U_{i j} \otimes|i\rangle\left\langle\left. j\right|_{\mathcal{T}},\right.
$$

where the $U_{i j}$, with $i, j=0,1$, act on $\mathcal{M}$. With this notation, condition (12) requires $U_{01}^{\dagger}|\psi\rangle_{\mathcal{M}}=0$, i.e., the operator $U_{01}$ must be singular. Therefore, if Alice and Bob select a unitary operation $U_{\mathcal{E}}$ such that $U_{01}$ is nonsingular, then $P_{f}<1$ for any pure state prepared by Eve.

What if Eve prepares a general mixed state $\rho_{\mathcal{E}}^{E}$ ? Any mixed state in $\mathcal{E}$ can be spectrally decomposed as $\rho_{\mathcal{E}}^{E}=$ $\sum_{i=0}^{3} \lambda_{i}\left|\phi_{i}\right\rangle\left\langle\left.\phi_{i}\right|_{\mathcal{E}}\right.$, where $0 \leq \lambda_{i} \leq 1, \sum_{i=0}^{3} \lambda_{i}=1$, and $\left\langle\phi_{i} \mid \phi_{j}\right\rangle_{\mathcal{E}}=\delta_{i j}, i, j=0, \cdots, 3$. When this decomposition of $\rho_{\mathcal{E}}^{E}$ is used in (8) and (9), these equations are transformed into a problem equivalent to the one posed by equations (10) and (11). Therefore, also in this more general case we can assure the result $P_{f}<1$. In fact, we can further show that, with the appropriate selection of $U_{\mathcal{E}}, P_{f}$ can be made at most $1 / 2$. As we have seen, $P_{f}$ can be written as

$$
P_{f}=\left\langle 0\left|\operatorname{tr}_{\mathcal{M}}\left(\rho_{\mathcal{E}}^{d}\right)\right| 0\right\rangle_{\mathcal{T}},
$$

with $\rho_{\mathcal{E}}^{d}=\left(\rho_{\mathcal{E}}^{E}+U_{\mathcal{E}}^{\dagger} \rho_{\mathcal{E}}^{E} U_{\mathcal{E}}\right) / 2$. Defining the projector $P=|00\rangle\left\langle\left. 00\right|_{\mathcal{E}}+\mid 10\right\rangle\left\langle\left. 10\right|_{\mathcal{E}}, P_{f}=\operatorname{tr}\left(\rho_{\mathcal{E}}^{d} P\right)\right.$. Using the properties of the trace operator,

$$
P_{f}=\operatorname{tr}\left(\rho_{\mathcal{E}}^{E} Q\right) / 2,
$$

where $Q=U_{\mathcal{E}} P U_{\mathcal{E}}^{\dagger}+P$ is a positive operator known to Eve, and with maximum eigenvalue $\lambda_{\max } \geq 1$. Therefore, the maximizing $\rho_{\mathcal{E}}^{E}$ is any eigenvector corresponding to $\lambda_{\max }$, and thus $P_{f}=\lambda_{\max } / 2$. Finally, it is easy to see (see, e.g., 16]) that choosing $U_{\mathcal{E}}$ such that it takes $P$ to its orthogonal complement makes $\lambda_{\max }=1$, and then, as predicted, $P_{f}=1 / 2$.

\section{B. Message attack}

This is a more subtle and severe family of attacks. Instead of directly forging a quantum message and send it to Bob, Eve could wait for Alice's message and manipulate it. Proceeding this way she tries to convert authentic messages into others with high probability of passing Bob's test.

In order to simplify the analysis, and without loss of generality, we shall distinguish between two types of message attacks. In the first one, Eve tries to extract information, by means of the appropriate measurement of the message in the channel, that allows her to prepare a different message that Bob regards as authentic. In the second class of attacks, Eve does not care about the current message in the channel. Instead, based on the knowledge of all the public aspects of the quantum authentication scheme used, she determines a quantum operation and applies it to any data sent by Alice. This quantum operation can be described by a TPCP map.

\section{Measurement}

According to equation (1), the information to which Eve has access is

$$
\rho_{\mathcal{E}}^{e}=\frac{1}{2}\left[\rho _ { \mathcal { M } } \otimes | 0 \rangle \left\langle\left.0\right|_{\mathcal{T}}+U_{\mathcal{E}}\left(\rho_{\mathcal{M}} \otimes|0\rangle\left\langle\left. 0\right|_{\mathcal{T}}\right) U_{\mathcal{E}}^{\dagger}\right] .\right.\right.
$$

Since Eve knows how the protocol works, she could get information about the key if, performing the appropriate measurement on the channel, she could perfectly distinguish between the two terms on the right-hand side of (16). If Eve managed to achieve it, she would collapse the state of Alice and Bob shared-key in a known unentangled pure quantum state, so she could throw away the authentic message and prepare and send to Bob a new one that would pass his test. Because Eve does not know which quantum message, $\rho_{\mathcal{M}}$, has been sent, the only way to discern between the two terms is by means of their tag-portions. Therefore, in order to make this strategy successful, the states $|0\rangle\left\langle\left. 0\right|_{\mathcal{T}}\right.$ and $\operatorname{tr}_{\mathcal{M}}\left[U_{\mathcal{E}}\left(\rho_{\mathcal{M}} \otimes|0\rangle\left\langle\left. 0\right|_{\mathcal{T}}\right) U_{\mathcal{E}}^{\dagger}\right]\right.$ must be perfectly distinguishable, i.e.,

$$
\left\langle0 \left|\operatorname { t r } _ { \mathcal { M } } \left[ U_{\mathcal{E}}\left(\rho_{\mathcal{M}} \otimes|0\rangle\left\langle\left. 0\right|_{\mathcal{T}}\right) U_{\mathcal{E}}^{\dagger}\right]|0\rangle_{\mathcal{T}}=0 .\right.\right.\right.
$$

Making use of the spectral decomposition of $\rho_{\mathcal{M}}$ (see our reasoning in Section III A), the requirement above can be alternatively written, without loss of generality, as

$$
\operatorname{tr}_{\mathcal{M}}\left(U_{\mathcal{E}}|\phi\rangle\left\langle\left.\phi\right|_{\mathcal{M}} \otimes \mid 0\right\rangle\left\langle\left. 0\right|_{\mathcal{T}} U_{\mathcal{E}}^{\dagger}\right)=|1\rangle\left\langle\left. 1\right|_{\mathcal{T}},\right.\right.
$$

where $|\phi\rangle_{\mathcal{M}}$ is any state in $\mathcal{M}$. Equation (18) is satisfied when $U_{\mathcal{E}}\left(|\phi\rangle_{\mathcal{M}} \otimes|0\rangle_{\mathcal{T}}\right)=|\omega\rangle_{\mathcal{M}} \otimes|1\rangle_{\mathcal{T}}$, where $|\omega\rangle_{\mathcal{M}}$ is 
some state in $\mathcal{M}$. Following a procedure parallel to the one employed in Section III A, we obtain the requirement that $U_{00}$ must be singular. Therefore, if Alice and Bob select an operation $U_{\mathcal{E}}$ such that $U_{00}$ is nonsingular, Eve cannot infer from her measurement the necessary information about the key.

\section{TPCP map}

Consider that Alice sends to Bob a quantum taggedmessage $\rho_{\mathcal{E}}$. If no eavesdropping takes place, the state in the channel is given by (4). But, assume now that Eve has the power to perform an arbitrary TPCP map, $\$$, on the tagged-message sent. Eve wants to choose $\$$ such that the decoding procedure performed by Bob on the resulting state led to any state not equal to $\rho_{\mathcal{M}}$ while the tag remains in the state $|0\rangle_{\mathcal{T}}$.

The global state resulting from Bob's decoding operation after Eve's TPCP mapping is $\rho_{A B \mathcal{E}}^{d}=$ $D_{B \mathcal{E}} \rho_{A B \mathcal{E}}^{E} D_{B \mathcal{E}}^{\dagger}$, where $\rho_{A B \mathcal{E}}^{E}=\$\left(\rho_{A B \mathcal{E}}^{e}\right)$, with $\rho_{A B \mathcal{E}}^{e}$ given by (3). The tagged-message decoded by Bob is

$$
\rho_{\mathcal{E}}^{d}=\operatorname{tr}_{A B}\left(\rho_{A B \mathcal{E}}^{d}\right)=\frac{1}{2}\left[\$\left(\rho_{\mathcal{E}}\right)+U_{\mathcal{E}}^{\dagger} \$\left(U_{\mathcal{E}} \rho_{\mathcal{E}} U_{\mathcal{E}}^{\dagger}\right) U_{\mathcal{E}}\right]
$$

The probability of Bob's accepting the message as a valid one is $P_{T P C P}=\left\langle 0\left|\operatorname{tr}_{\mathcal{M}}\left(\rho_{\mathcal{E}}^{d}\right)\right| 0\right\rangle_{\mathcal{T}}$. Clearly, this probability is one if and only if $\operatorname{tr}_{\mathcal{M}}\left(\rho_{\mathcal{E}}^{d}\right)=|0\rangle\left\langle\left. 0\right|_{\mathcal{T}}\right.$, which means that the conditions

$$
\begin{aligned}
\operatorname{tr}_{\mathcal{M}}\left[\$\left(\rho_{\mathcal{E}}\right)\right] & =|0\rangle\left\langle\left. 0\right|_{\mathcal{T}},\right. \\
\operatorname{tr}_{\mathcal{M}}\left[U_{\mathcal{E}}^{\dagger} \$\left(U_{\mathcal{E}} \rho_{\mathcal{E}} U_{\mathcal{E}}^{\dagger}\right) U_{\mathcal{E}}\right] & =|0\rangle\left\langle\left. 0\right|_{\mathcal{T}},\right.
\end{aligned}
$$

must be simultaneously satisfied. In order to check whether Eve succeeds, let us first consider the most simple case of TPCP, that in which she performs a unitary operation $A_{\mathcal{E}}$. Using again the spectral decomposition of $\rho_{\mathcal{M}}$ (see, again, our reasoning in Section III A), the conditions above can be alternatively written as

$$
\begin{aligned}
\operatorname{tr}_{\mathcal{M}}\left[A_{\mathcal{E}}\left(|\phi\rangle\left\langle\left.\phi\right|_{\mathcal{M}} \otimes \mid 0\right\rangle\left\langle\left. 0\right|_{\mathcal{T}}\right) A_{\mathcal{E}}^{\dagger}\right]\right. & =|0\rangle\left\langle\left. 0\right|_{\mathcal{T}},\right. \\
\operatorname{tr}_{\mathcal{M}}\left[B_{\mathcal{E}}\left(|\phi\rangle\left\langle\left.\phi\right|_{\mathcal{M}} \otimes \mid 0\right\rangle\left\langle\left. 0\right|_{\mathcal{T}}\right) B_{\mathcal{E}}^{\dagger}\right]\right. & =|0\rangle\left\langle\left. 0\right|_{\mathcal{T}},\right.
\end{aligned}
$$

where $|\phi\rangle_{\mathcal{M}}$ is any state in $\mathcal{M}$, and $B_{\mathcal{E}}=U_{\mathcal{E}}^{\dagger} A_{\mathcal{E}} U_{\mathcal{E}}$. Then, it is straightforward to see that, in order to fulfill (22) and (23), Eve needs to choose a unitary operation such that

$$
\begin{aligned}
& A_{\mathcal{E}}=\sum_{i=0}^{1} A_{i i} \otimes|i\rangle\left\langle\left. i\right|_{\mathcal{T}},\right. \\
& B_{\mathcal{E}}=\sum_{i=0}^{1} B_{i i} \otimes|i\rangle\left\langle\left. i\right|_{\mathcal{T}},\right.
\end{aligned}
$$

where the $A_{i i}$ and $B_{i i}, i=0,1$, are some unitary operations on the state space $\mathcal{M}$. Since $B_{\mathcal{E}}$ and $A_{\mathcal{E}}$ are related by $B_{\mathcal{E}}=U_{\mathcal{E}}^{\dagger} A_{\mathcal{E}} U_{\mathcal{E}}$, we can arrange equations (24) and (25) in matrix form and write

$$
\left(\begin{array}{cc}
U_{00}^{\dagger} & U_{10}^{\dagger} \\
U_{01}^{\dagger} & U_{11}^{\dagger}
\end{array}\right) \cdot\left(\begin{array}{cc}
A_{00} & 0 \\
0 & A_{11}
\end{array}\right) \cdot\left(\begin{array}{ll}
U_{00} & U_{01} \\
U_{10} & U_{11}
\end{array}\right)=\left(\begin{array}{cc}
B_{00} & 0 \\
0 & B_{11}
\end{array}\right) .
$$

According to our formulation of the problem, Eve would succeed in her attack if, given the unitary operator $U_{\mathcal{E}}$, she could always find two unitary operators $A_{\mathcal{E}}$ and $B_{\mathcal{E}}$ such that $U_{\mathcal{E}} B_{\mathcal{E}}=A_{\mathcal{E}} U_{\mathcal{E}}$. Of course, the trivial solution $A_{\mathcal{E}}=B_{\mathcal{E}}=I_{\mathcal{E}}$ (Eve does not modify the state of the tagged-message) is discarded.

In Section III A we have shown that Alice and Bob can avoid Eve's forgering of messages if $U_{01}$ is nonsingular. In a similar way, in Section III B 1 we have seen that Alice and Bob can prevent Eve from gaining critical information about the key by way of measurement if $U_{00}$ is also nonsingular. If we assume now that these conditions hold, i.e., that $U_{00}$ and $U_{01}$ are nonsingular, then it can be shown, using the unitarity of $U_{\mathcal{E}}$, that $U_{10}$ and $U_{11}$ are then also nonsingular. Under these conditions, as we show in Appendix A, the equation $U_{\mathcal{E}} B_{\mathcal{E}}=A_{\mathcal{E}} U_{\mathcal{E}}$ has always (for any $U_{\mathcal{E}}$ ) the prescripted solution, and Eve can always be successful in her attack. This unconditional success of Eve's attack makes unnecessary to consider the more general case of a TPCP map.

\section{Discussion on the structure of the tag space}

Let us now briefly explain why increasing the dimension of the tag space does not affect any of the arguments considered in this section.

As seen by Eve, the key shared by Alice and Bob controls whether the quantum state in the channel belongs to the two-dimensional subspace spanned by the vectors $\left\{|0\rangle_{\mathcal{M}}|0\rangle_{\mathcal{T}},|1\rangle_{\mathcal{M}}|0\rangle_{\mathcal{T}}\right\}$ or to the one spanned by $\left\{U_{\mathcal{E}}\left(|0\rangle_{\mathcal{M}}|0\rangle_{\mathcal{T}}\right), U_{\mathcal{E}}\left(|1\rangle_{\mathcal{M}}|0\rangle_{\mathcal{T}}\right)\right\}$, so there are two alternative coding subspaces for the valid quantum messages. All the conditions the unitary operator $U_{\mathcal{E}}$ has to fulfill in order to avoid Eve's attacks can be geometrically interpreted as conditions on the relative position between these two code subspaces of the four-dimensional space $\mathcal{E}$. For instance, in order to avoid the success of the nomessage attack, as stated by the conditions (8)-(9), the subspaces must $\operatorname{span} \mathcal{E}$. On the other hand, in order to avoid the measurement attack, condition (17), both subspaces must be non-orthogonal. As for the unitary attack, we have seen that Eve can always fulfill conditions (24)-(25), i.e. she can always find a unitary operator acting on $\mathcal{E}$ such that it leaves invariant both subspaces at the same time, independently of their relative position, and rotate the vectors within in a non trivial way.

If the dimension of $\mathcal{T}$ is increased, the dimension of the space $\mathcal{E}$ also increases (say to $N$, with $N>4$ ), 
but if the valid tag state is still some particular state $|\mathbf{0}\rangle_{\mathcal{T}} \equiv|0 \cdots 0\rangle_{\mathcal{T}}$, the dimension of the two alternative code subspaces will remain equal to two. Thus we are just embedding the problem in a larger space, but not changing its intrinsic complexity: In a $N$-dimensional space Alice and Bob would still be able to choose two two-dimensional code subspaces neither linearly dependent nor orthogonal, and Eve would still find a unitary nontrivial operator in $U(N)$ whose restriction to the particular four-dimensional space containing the two codes leaves both codes invariant.

One may also consider what would happen if we allowed the tag state to be a mixed, $\rho_{\mathcal{T}}$, rather than a pure state. According to our protocol, in order to have perfect deterministic decoding, the tag should belong to any subspace of the tag space (so that Alice an Bob could perfectly distinguish between valid or invalid messages). When measuring a mixed state one can obtain any state belonging to the subspace the mixed state has support on (with a certain probability, given by the spectral decomposition). Consequently, when Bob verifies the tag, he will consider as valid any result giving a state inside that subspace, and will discard the message if the result lays in the orthogonal complement. Since the dimension of the two distinct code subspaces increases, things are slightly more complicated when the tag is not pure. Let $n<N$ be the dimension of the valid code subspaces, and let us see whether we can generalize conditions (8)-(9) and (17)-(18).

Generalization of conditions (8)-(9) above is achieved if and only if, for some state $\rho_{\mathcal{E}}^{E}$, the states $\operatorname{tr}_{\mathcal{M}}\left(\rho_{\mathcal{E}}^{E}\right)$ and $\operatorname{tr}_{\mathcal{M}}\left(U_{\mathcal{E}}^{\dagger} \rho_{\mathcal{E}}^{E} U_{\mathcal{E}}\right)$ belong to the valid tag subspace. But, as argued above, this can be avoided by Alice and Bob choosing an operator $U_{\mathcal{E}}$ such that the block of $U_{\mathcal{E}}^{\dagger}$ transforming states from the valid tag subspace to the invalid one (the analogous to $U_{01}^{\dagger}$ in $(13)$ ) is nonsingular. Note that if $n<N / 2$ this block is not a square operator, and nonsingular means with trivial kernel. Note also that choosing $n>N / 2$ (a subspace of valid messages bigger than the invalid one) is insecure, since there would always be vectors of the valid subspace transformed into the null state by that block, and so Eve would be successful in the no-message attack with probability one.

Generalization of equations (17)-(18), related to the measurement attack, is achieved if and only if the state $\operatorname{tr}_{\mathcal{M}}\left[U_{\mathcal{E}}\left(\rho_{\mathcal{M}} \otimes \rho_{\mathcal{T}}\right) U_{\mathcal{E}}^{\dagger}\right]$ belongs to the invalid tag subspace. But again this can be avoided choosing $U_{\mathcal{E}}$ such that the equivalent to block $U_{00}$ (now a $n^{2}$-dimensional block operator, transforming states within the valid tag subspace) is nonsingular.

But the crucial fact is that Eve can still fulfill conditions (24)-(25), i.e., there is always a nontrivial unitary operator leaving invariant the two code subspaces. To see this, note that the generalization of equations (20)(23) is obtained by simply imposing that the states in the left-hand side of those equations belong to the valid tag subspace. Then equations (24)-(26) would have the same form, apart from the fact that now $A_{00}, B_{00}$ and $U_{00}$ would represent $n^{2}$-dimensional operators, $A_{11}, B_{11}$ and $U_{11}(N-n)^{2}$-dimensional ones, $U_{01}$ a $n \times(N-n)$ operator, and $U_{10}$ a $(N-n) \times n$ one. The solutions for $A_{00}$ and $A_{11}$ given in the appendix equally hold if $n=N / 2$ (equations (A4)- A5) are exactly the same, all the blocks are square operators, and we can invert them). If $n<N / 2$ then we are just embedding a $2 n<N$ problem into a larger $N$-dimensional space, and thus the solution of the intrinsic problem still exists.

The fact that increasing the dimension of $\mathcal{T}$ does not improve the robustness of the protocol may surprise the reader familiar with Quantum Error Correction Codes (QECC). In these codes more qubits of tag are added to protect against more errors. But note that the nature of the errors considered is statistical, i.e. they are randomly generated by the noise in the channel. In QECC the efficiency of the correction capability lays on the assumption that statistical errors on a large number of qubits are less likely than those on a small number. The errors considered here, arbitrary unitary actions on the space $\mathcal{E}$, do not belong to this category.

\section{CONCLUSION}

Providing message authentication is one of the main goals of communication security. Classical messageauthentication methods can be combined with quantum teleportation to make the authentication of quantum information possible. However, it is not yet clear whether this procedure is optimal in the resources it requires. In this paper we study the authentication of elementary quantum messages (qubits) using minimum-size keys. Specifically, we have generalized a previous class of quantum authentication protocols 12] to the case of one-qubit messages, and studied its security against a forger with quantum power and full access to the channel between the communicating parties. The main result of this study is that, unlike classical binary messages, the intrinsic nature of quantum information makes the authentication of one qubit using a minimal key impossible, i.e. it is not possible to keep the failure probability of the qubit authentication protocol below one. We are currently investigating the minimum amount of quantum resources needed to authenticate a qubit, and the use of more general quantum operations (TPCP maps) in the encoding and decoding actions. Our results will be published elsewhere.

\section{Acknowledgments}

The authors acknowledge Howard Barnum for sharing with them his early work on quantum message authentication codes, and Adán Cabello, Debbie Leung, and an anonymous referee for their insightful comments. We gratefully thank some members of the Applied Math- 
ematics Department at the University of Vigo, particularly, I. Area and A. Martín, as well as R. Israel, from the University of British Columbia, for their assistance with Matrix Analysis. This work was par- tially supported by Xunta de Galicia (Spain, grant n. PGIDT00PXI322060PR) and the Spanish Government (grant n. TIC2001-3217).
[1] R. L. Rivest, Handbook of Theoretical Computer Science, Volume A: Algorithms and Complexity (Elsevier and MIT Press, 1990), chap. 13. Cryptography, pp. 717755.

[2] A. J. Menezes, P. C. V. Oorschot, and S. A. Vanstone, Handbook of Applied Cryptography, Series on Discrete Mathematics and Its Applications (CRC Press, 1996).

[3] M. N. Wegman and J. L. Carter, J. Computer and System Sciences 22, 265 (1981).

[4] W. Diffie and M. E. Hellman, IEEE Transactions on Information Theory IT-22, 644 (1976).

[5] R. L. Rivest, A. Shamir, and L. M. Adleman, Communications of the ACM 21(2), 120 (1978).

[6] D. W. Kravitz, Digital signature algorithm, U. S. Patent \# 5,231,668 (27 July 1993).

[7] P. W. Shor, SIAM J. Computing 26, 1484 (1997).

[8] D. Ljunggren, M. Bourennane, and A. Karlsson, Physical Review A 62, 022305 (2000).

[9] G. Zeng and W. Zhang, Physical Review A 61, 022303 (2000).

[10] D. Gottesman and I. Chuang, Quantum digital signatures, quant-ph/0105032.

[11] D. W. Leung, Quantum Vernam cipher, quant$\mathrm{ph} / 0012077$.

[12] M. Curty and D. J. Santos, Physical Review A 64, 062309 (2001).

[13] M. A. Nielsen and C. M. Caves, Physical Review A 55, 2547 (1997).

[14] M. A. Nielsen, C. M. Caves, B. Schumacher, and H. Barnum, Proc. R. Soc. Lond. A 454, 277 (1998).

[15] C. M. Caves, Journal of Superconductivity 12(6), 707 (1999).

[16] R. A. Horn and C. R. Johnson, Matrix Analysis (Cambridge University Press, Cambridge, 1985).

\section{APPENDIX A}

In this appendix we show that, given a unitary operator $U_{\mathcal{E}}$, acting on a four-dimensional space $\mathcal{E}=\mathcal{M} \otimes \mathcal{T}$, and with the $2 \times 2$ blocks of its matrix representation nonsingular, one (Eve) can always find two unitary, block-diagonal operators , $A_{\mathcal{E}}$ and $B_{\mathcal{E}}$, such that $U_{\mathcal{E}}^{\dagger} A_{\mathcal{E}} U_{\mathcal{E}}=B_{\mathcal{E}}$.

The explicit expressions for the operators in their block form can be written as:

$$
\begin{array}{r}
U_{\mathcal{E}}=\sum_{i=0}^{1} \sum_{j=0}^{1} U_{i j} \otimes|i\rangle\left\langle\left. j\right|_{\mathcal{T}},\right. \\
A_{\mathcal{E}}=\sum_{i=\overline{1}^{0}}^{1} A_{i i} \otimes|i\rangle\left\langle\left. i\right|_{\mathcal{T}},\right. \\
B_{\mathcal{E}}=\sum_{i=0} B_{i i} \otimes|i\rangle\left\langle\left. i\right|_{\mathcal{T}} .\right.
\end{array}
$$

From the equality of the blocks resulting from multiplying by $|0\rangle\left\langle\left. 1\right|_{\mathcal{T}}\right.$ and $\left.\mid 1\right\rangle\left\langle\left. 0\right|_{\mathcal{T}}\right.$ both sides of $U_{\mathcal{E}}^{\dagger} A_{\mathcal{E}} U_{\mathcal{E}}=B_{\mathcal{E}}$, we obtain the equations:

$$
\begin{aligned}
& U_{10}^{\dagger} A_{11} U_{11}+U_{00}^{\dagger} A_{00} U_{01}=0 \\
& U_{11}^{\dagger} A_{11} U_{10}+U_{01}^{\dagger} A_{00} U_{00}=0 .
\end{aligned}
$$

Since all the $U_{i j}$ blocks are invertible, we have the following two expressions for $A_{11}$ :

$$
\begin{aligned}
A_{11} & =-U_{10}^{\dagger^{-1}} U_{00}^{\dagger} A_{00} U_{01} U_{11}^{-1} \\
& =-U_{11}^{\dagger^{-1}} U_{01}^{\dagger} A_{00} U_{00} U_{10}^{-1}
\end{aligned}
$$

The second equality in this equation implies: $A_{00} U_{01} U_{11}^{-1} U_{10} U_{00}^{-1}=U_{00}^{\dagger^{-1}} U_{10}^{\dagger} U_{11}^{\dagger^{-1}} U_{01}^{\dagger} A_{00}$, that is, $A_{00} G=G^{\dagger} A_{00}$, with $G=U_{01} U_{11}^{-1} U_{10} U_{00}^{-1}$. Using the following relation between the $U_{i j}$ blocks, derived from the unitarity of $U_{\mathcal{E}}$,

$$
U_{00}^{\dagger} U_{01}+U_{10}^{\dagger} U_{11}=0
$$

we can write $G=-U_{00}^{-1^{\dagger}} U_{10}^{\dagger} U_{10} U_{00}^{-1}$, so $G$ is hermitian, and, therefore, $A_{00}$ and $G$ commute. Any hermitian operator commutes with some unitary operator that is not a scalar multiple of the identity, so this guarantees $A_{00}$ exists. Given $A_{00}$, we can obtain $A_{11}$ from (A6). Now it remains to be shown that such $A_{11}$ is also unitary. Computing the adjoint of the first expression in (A6) and multiplying it by the second, we obtain:

$$
A_{11}^{\dagger} A_{11}=U_{11}^{\dagger^{-1}} U_{01}^{\dagger} A_{00}^{\dagger} U_{00} U_{10}^{-1} U_{11}^{\dagger^{-1}} U_{01}^{\dagger} A_{00} U_{00} U_{10}^{-1} .
$$

From (A7), $U_{11}^{\dagger} U_{10}=-U_{01}^{\dagger} U_{00}$. Applying this result to the equation above leads to

$$
\begin{aligned}
A_{11}^{\dagger} A_{11} & =-U_{11}^{-1^{\dagger}} U_{01}^{\dagger} A_{00}^{\dagger} A_{00} U_{00} U_{10}^{-1} \\
& =-U_{11}^{-1^{\dagger}} U_{01}^{\dagger} U_{00} U_{10}^{-1}=U_{11}^{-1^{\dagger}} U_{11} U_{10} U_{10}^{-1}=I,
\end{aligned}
$$

and, therefore, $A_{11}$ is unitary.

As for $B_{00}$ and $B_{11}$, they can be obtained, in terms of $A_{00}$ and $A_{11}$, from the equality between the blocks of $U_{\mathcal{E}}^{\dagger} A_{\mathcal{E}} U_{\mathcal{E}}=B_{\mathcal{E}}$, when both of its sides are multiplied by $|0\rangle\left\langle\left. 0\right|_{\mathcal{T}}\right.$ and $\left.\mid 1\right\rangle\left\langle\left. 1\right|_{\mathcal{T}}\right.$. Their unitarity is proven in a similar way. 